\title{
Specifications of Resonant Acoustic Rotating Waves in Circular and Annular Domains
}

\author{
EUGENE SVIYAZHENINOV \\ Institute for Problems in Mechanical Engineering of Russian Academy of Sciences \\ Bolshoy pr. V.O., 61, St.Petersburg, 199178 \\ RUSSIA \\ sviage@gmail.com
}

\begin{abstract}
Circular and annular domains of hydroacoustic vibration are very common in modern technology due to their simplicity. On the other hand it turns out that such a shape possesses remarkable vibration properties. It is determined that there are two classes of resonant rotating waves, predominantly tangential and predominantly radial, in terms of prevalence of tangential or radial components of the vectors of vibrational velocities and displacements. The complete map of resonant angular velocities shows that all predominantly tangential angular velocities for all values of ring thickness are assembled into the self-isolating unique single low-frequency branch, whereas predominantly radial ones fill the entire high-frequency region very densely.
\end{abstract}

Key-Words: - Hydroacoustic vibration, natural frequencies and forms, resonance

Received: November 7, 2019. Revised: February 5, 2020. Accepted: February 25, 2020. Published: March 16, 2020.

\section{Introduction}

In production of nano- and ultra-disperse inorganic powders from aqueous and nonaqueous solutions the so-called wet chemical methods are extensively distributed currently. The term "wet chemical methods" by itself has been deeply embedded into nanotechnology in order to emphasize the difference of these technique class from the ordinary solid-state methods of synthesizing compounds and materials, due to its principal feature: usage of a liquid phase at one of the process steps. The favorable distinctions of the wet chemistry products from analogous products of solid phase synthesis are substantially smaller grain or crystallite size and, as a rule, lower temperature and shorter duration of phase formation of multicomponent compounds. There are a lot of processes using a liquid phase. They are, for instance: liquid phase sol-gel process, hydrothermal synthesis, Pechini method, spray drying, aerosol spray pyrolysis, cryochemical synthesis and others [1]. Vibration technologies traditionally play an increasingly important part in manufacturing industry [2]. Usage of vibration in resonant regimes, providing optimal conditions for pumping and accumulation of vibrational energy, is especially effective. Resonant regimes are essencially favorable for hydroacoustic systems, since their working media - compressible gases or liquids, - do not suffer from prolonged intense loads, in contrast to solids subject to destruction. At resonance optimal conditions are created for pumping the energy of an external source into a mechanical oscillatory system with distributed parameters, because the vibrational velocities and pressures in the working medium become in-phase. So resonant dispersants and homogenizers are just the devices that should be widely used in the modern nanotechnology. A continuous steadystate mode of operation is most effectively implemented with rotational movement. That is why the research of resonant rotating waves is of the primary practical importance.

\section{Maps of dimensionless natural frequencies of hydroacoustic oscillations in a ring domain}

The discrete spectrum of the dimensionless natural frequencies of acoustic oscillations $\omega_{i k} \mathrm{R} / \mathrm{c}$, where $\mathrm{R}$ - the outer radius of the annular domain and $\mathrm{c}$ is the speed of sound of the gaseous or compressible fluid medium filling it, as a function of the ratio of the inner radius of the circular region to the outer radius $\rho, 0 \leq \rho<1$, is determined by the relations:

$\omega_{i k} \mathrm{R} / \mathrm{c}=\alpha_{i k}(\rho)$, 
where

$\alpha_{i k}(\rho)$ - the i-th root of the transcendental equation:

$\frac{J_{k}^{\prime}(\alpha \rho)}{J_{k}^{\prime}(\alpha)}=\frac{N_{k}^{\prime}(\alpha \rho)}{N_{k}^{\prime}(\alpha)}$,

$\mathrm{J}_{k}^{\prime}(\alpha \rho), \mathrm{N}_{k}^{\prime}(\alpha \rho)$ - are the derivatives of the k-th order Bessel and Neumann functions, respectively.

For the functions $\omega_{i k}(\rho), \alpha_{i k}(\rho)$, the first index $i$ denotes the ordinal number by increasing the root of equation (2) for a given value of $k$, whereas the second index $\mathrm{k}$ is the number of the nodal diameters of the eigenmode of the oscillations corresponding to the eigenfrequency $\omega_{i k}$. The indicated indices take the values: $i=1$, $2,3, \ldots ; \mathrm{k}=0,1,2,3, \ldots$, where $\mathrm{k}=0$ corresponds to an axisymmetric, or circumferentially nodefree natural form of vibrations, and $\mathrm{k}=1,2,3, \ldots$ - to a non-axisymmetric having nodal diameters eigenmodes.

All eigenfrequencies are shown in Fig. 4-7, while the lower ones, $\mathrm{i}=1$, nonaxisymmetric with successively increasing number of nodal diameters, $\mathrm{k}=1,2,3, \ldots$, predominantly tangential vibration eigenmodes, excited in resonant devices, for $\rho=.7,-$ in Fig. 8.

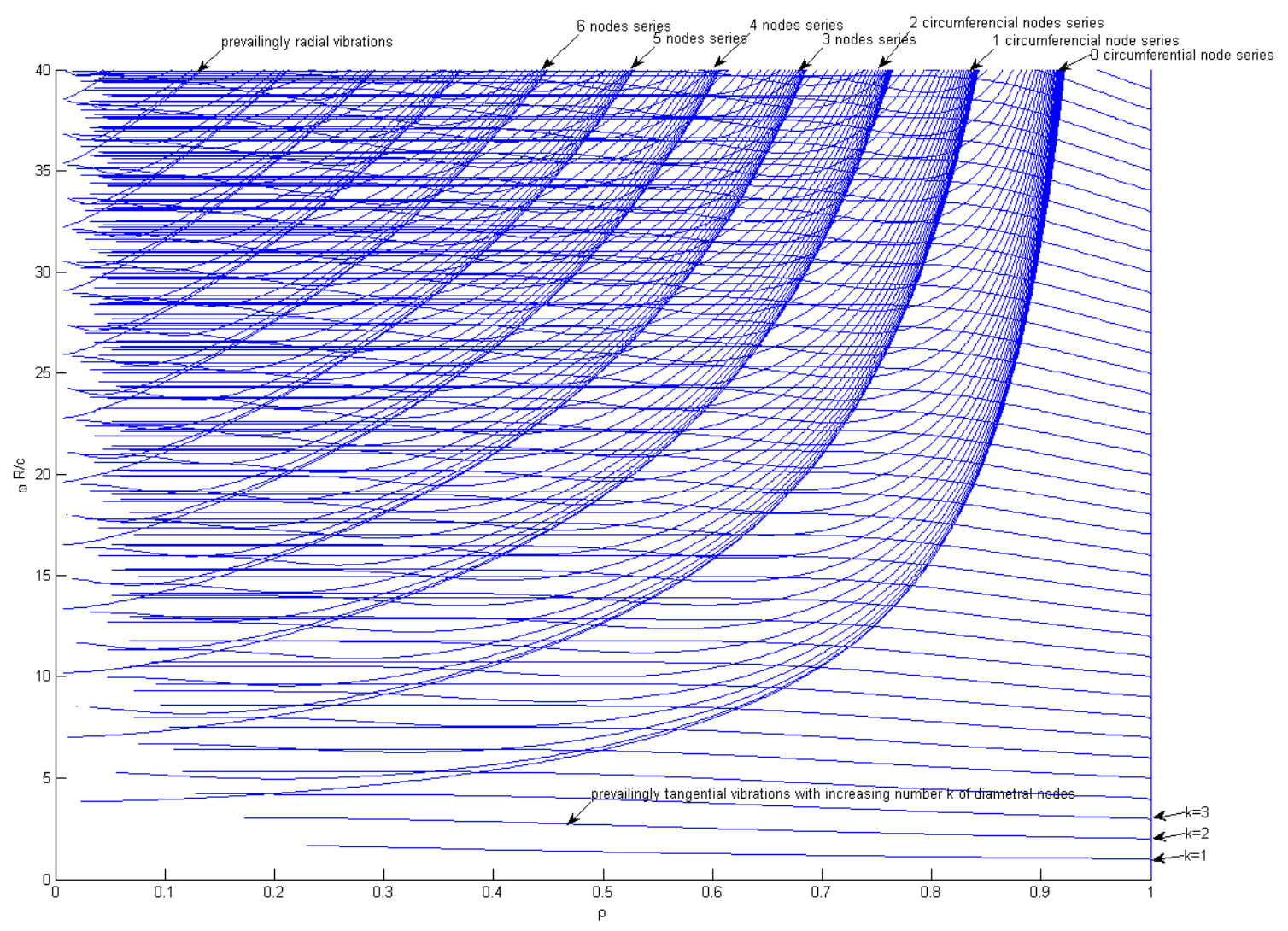

Figure 1. Small scale eigenfrequency map. 


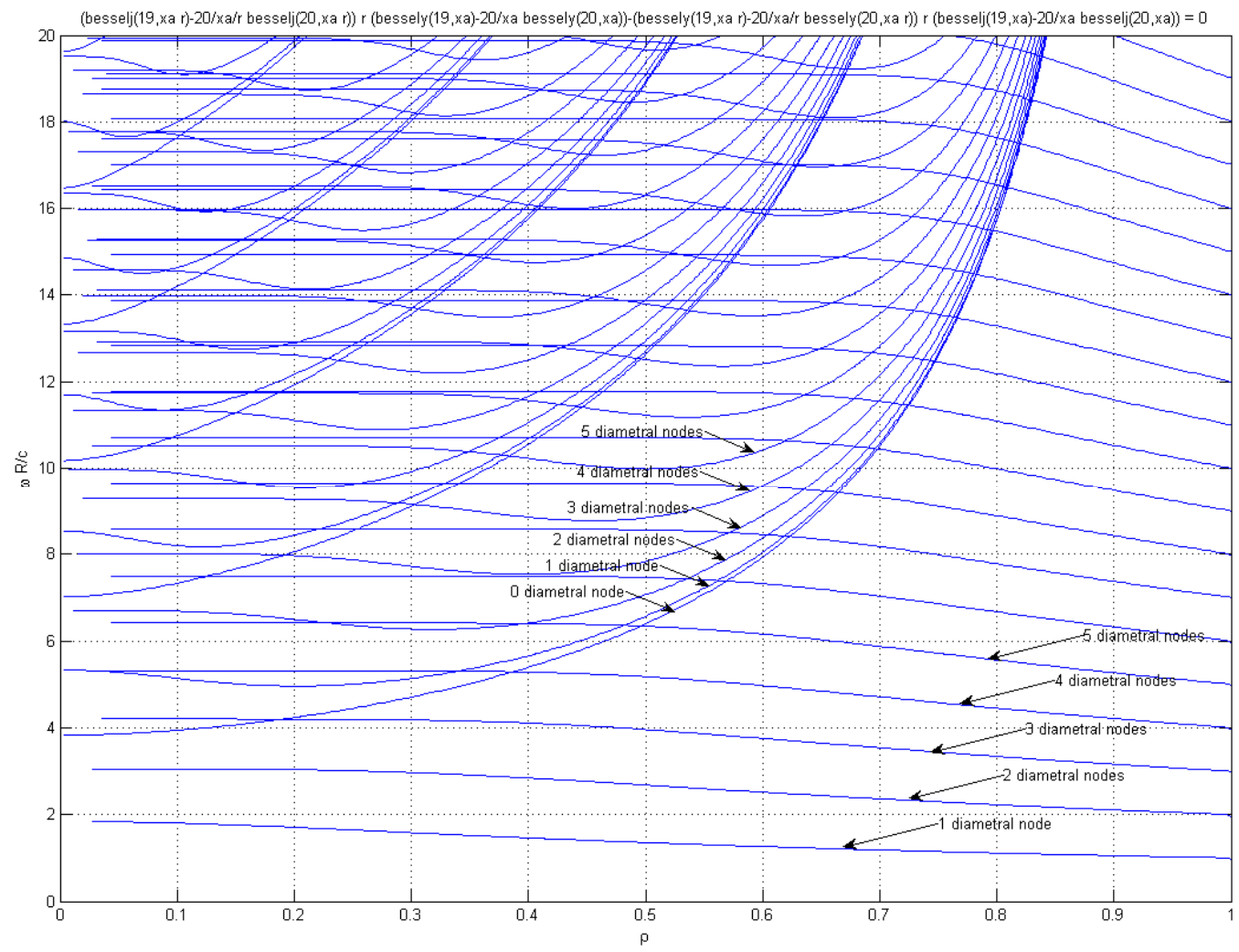

Figure 2. Medium scale eigenfrequency map.

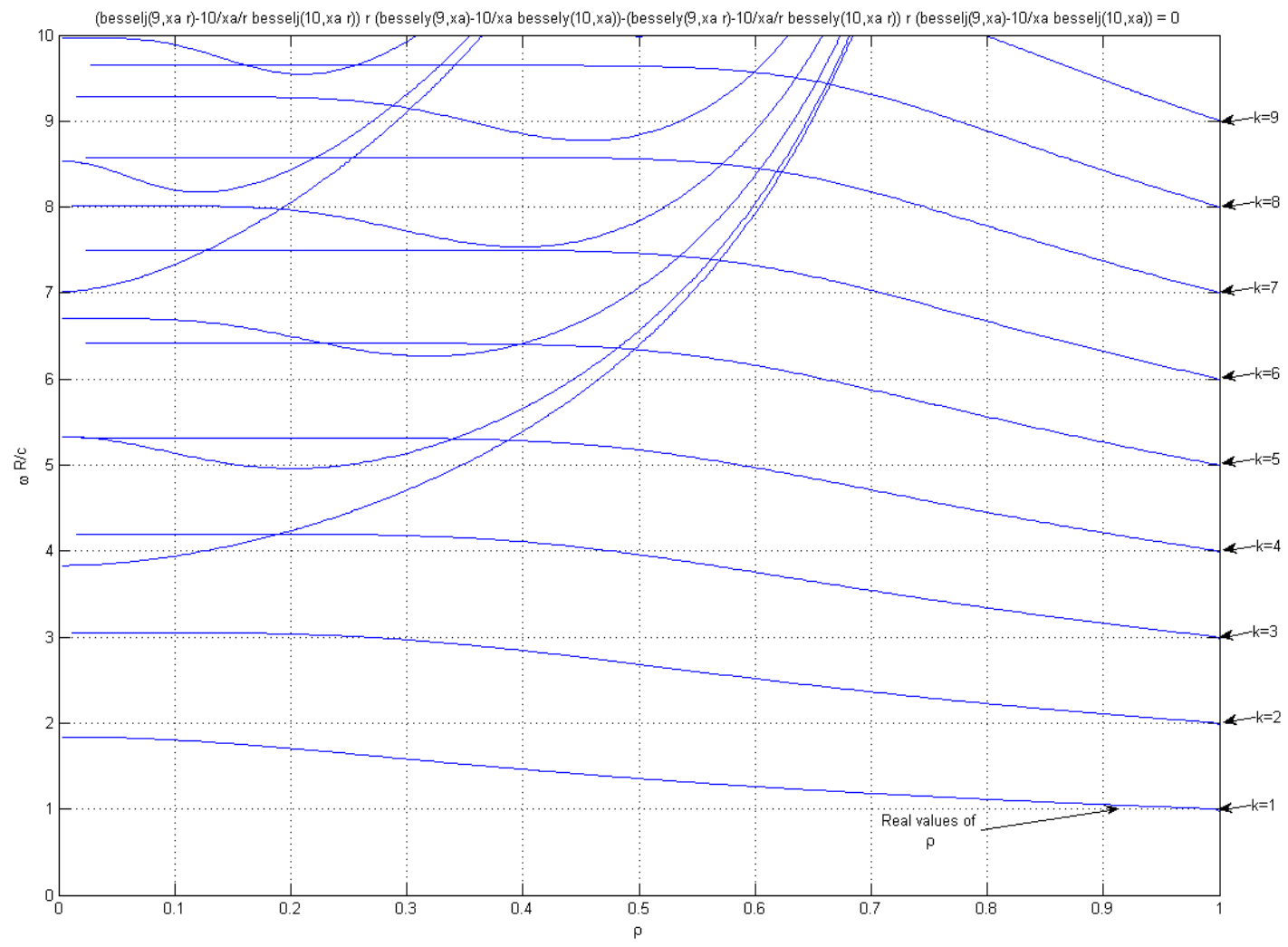

Figure 3. Large scale eigenfrequency map. 


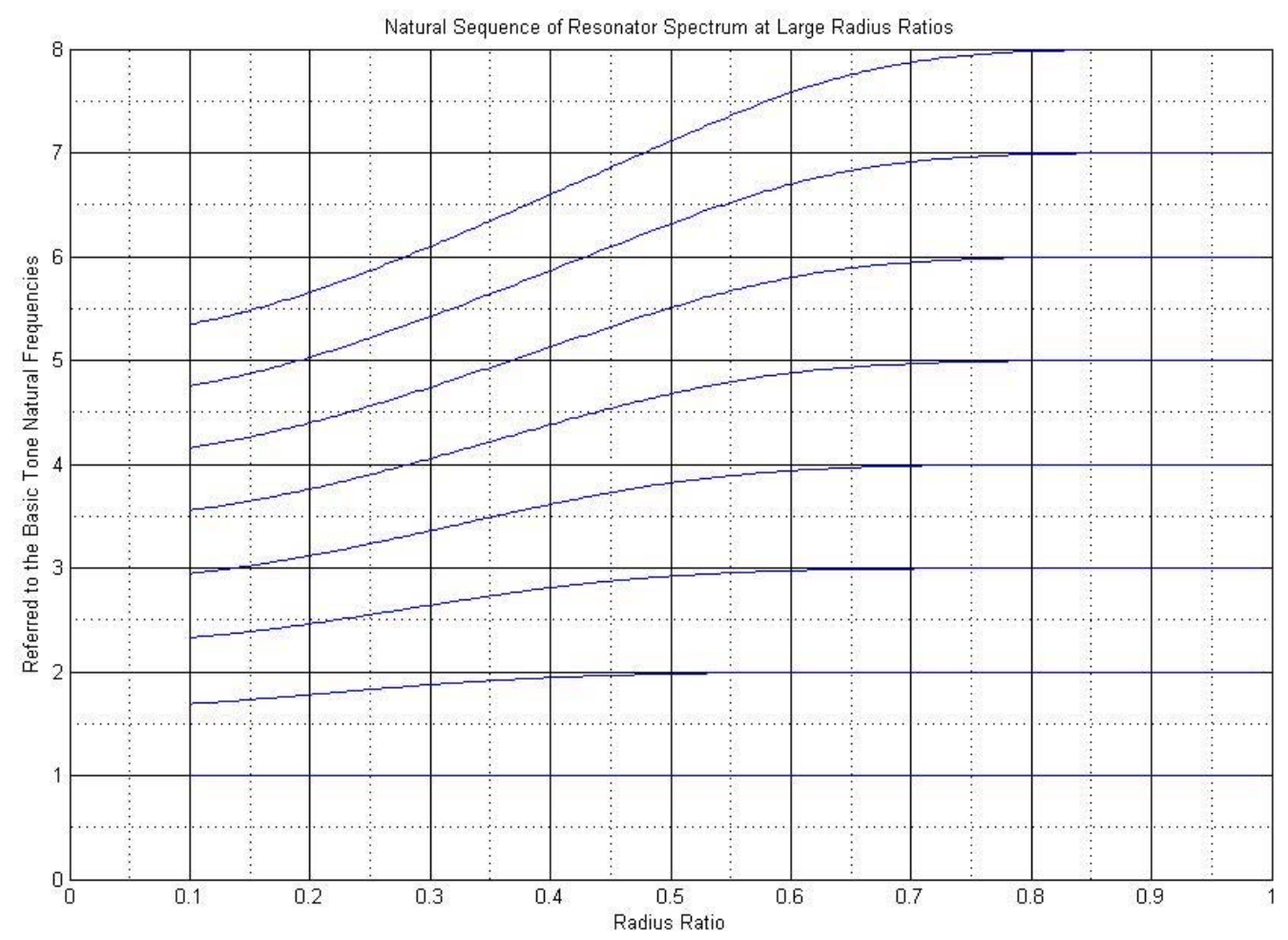

Figure 4. Eigenfrequencies referred to the fundamental frequency.

A comprehensive representation of the spectrum of natural frequencies of oscillations of an arbitrary thickness concentric region $0<\rho<1$, up to high-frequency ones, is provided with the complete map of natural frequencies (Fig. 1). It shows that two classes of natural oscillations are distinguished - predominantly tangential and predominantly radial, in terms of prevalence of the tangential or radial components of the vibrational velocity vectors.

The first class is formed by regular weakly decreasing with decreasing $\rho$ almost horizontal curves, at $\rho \rightarrow 1$ forming a strictly harmonic sequence in eigenfrequencies: $1,2,3$, $\ldots, \mathrm{k}, \ldots$ where $\mathrm{k}$ is a natural number. These are predominantly tangential vibrations without nodal circles, with a successively increasing number $\mathrm{k}$ of nodal diameters.

The second class is formed with predominantly radial vibrations. On the full natural frequency map (Fig. 1), it forms the series of "decreasing cluster families", each of which is bounded to the right by asymptotic curves. Each of these asymptotes corresponds to axisymmetric oscillations. The rightmost asymptote represents vibrations without nodal circles, the next to the left - with one nodal circle, and so on, with the addition of exactly one nodal circle when moving to the left on the map.
To analyze the composition of families of predominantly radial oscillations, it is necessary to turn to a larger-scale mid-frequency eigenfrequency map (Fig. 2). It can be seen how the frequency curves differing by successive addition of the next nodal diameter in the corresponding natural form of acoustic oscillations are successively adjacent to the left of each asymptote of the family.

And, finally, the low-frequency map of the natural frequencies of oscillations has the utmost significance for solution of the investigated problem (Fig. 3).

It exactly shows that with increasing angular velocity $\omega$ of the rotating acoustic load, this perturbation primarily excites the lowfrequency predominantly tangential eigenmodes, located on the frequency map in the lower righthand region corresponding to the real relative dimensions $\rho$ of the annular region. In this case, the exact equality for the dimensionless natural frequencies of the compressible gas in a resonant chamber is fulfilled for $\rho \rightarrow 1: \omega_{i k} \mathrm{R} / \mathrm{c}=\mathrm{k}$, where $\mathrm{i}=1 ; \mathrm{k}$ is a natural number having the physical meaning of the number of nodal diameters of the natural form of predominantly tangential oscillations (Fig. 5). The natural frequencies $\omega_{1 k}$ by themselves, related to the basic tone frequency $\omega_{11}$, that is, $\omega_{1 k} / \omega_{11}$, form a strict 
natural sequence for sufficiently large values of $\rho$ (Fig. 4). Thus, Fig. 4 shows that for $0.7<\rho<1$
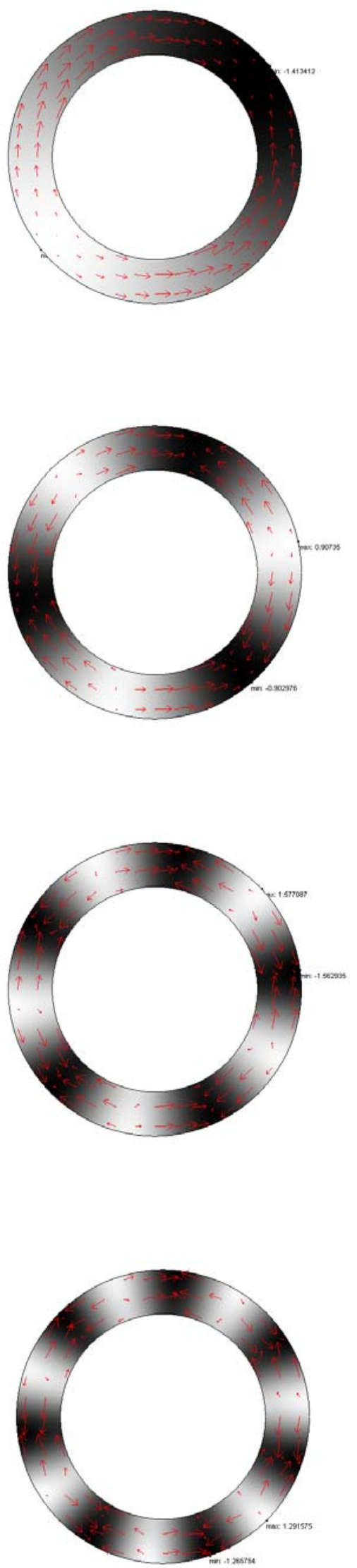

this natural sequence is satisfied for the eight lowest eigenfrequencies.
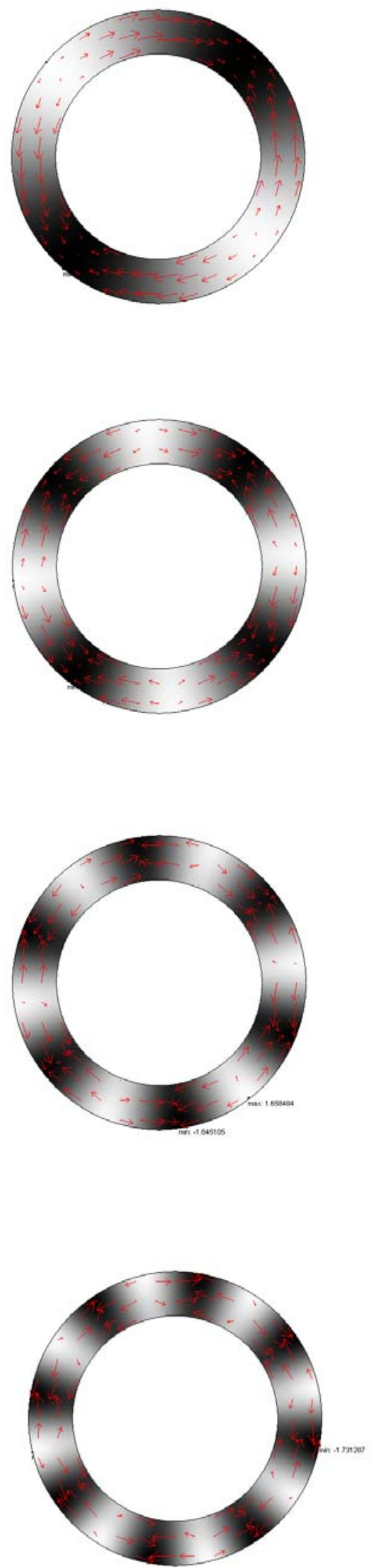

Figure 5. Predominantly tangential hydroacoustic vibration eigenmodes. 
Summing up the discussion of eigenfrequency maps (Fig. 1-4) and eigenmode diagrams (Fig. 5) of acoustic oscillations of annular chambers, we summarize that for large values of $\rho$, corresponding to the real dimensions of the chamber, predominantly tangential low frequency oscillations with nodal diameters only are located, whereas in the regions of small $\rho$, high-frequency predominantly radial acoustic oscillations with nodal circles also appear. Just predominantly tangential vibrations are used to generate rotating acoustic waves in the resonant device [3].

\section{Distinctive feature of circular geometric shape of a resonant chamber}

Each non-axisymmetric eigenmode $\mathrm{A}(\mathrm{r}) \operatorname{cosk} \theta$, $\mathrm{k}=1,2,3, \ldots$ (Fig. 5) is a solution of the equation of free acoustic oscillations and is interpreted by the standing wave:

$\mathrm{A}(\mathrm{r}) \operatorname{cosk} \theta \cos \omega_{i k} \mathrm{t}$,

where $r$ is the radial, $\theta$ is the angular coordinate, $\mathrm{t}$ is time, $\mathrm{k}$ is the number of nodal diameters, $\omega_{i k}$ is the corresponding natural frequency of oscillations. The distinguishing feature of the circular geometric shape of a resonant chamber is that all eigenfrequencies are doubly degenerate, i.e. each natural oscillation frequency $\omega_{i k}$, in addition to (3), corresponds to another non-axisymmetric eigenmode of oscillations $\mathrm{A}(\mathrm{r}) \operatorname{sink} \theta$, also interpreted by the standing wave:

$\mathrm{A}(\mathrm{r}) \operatorname{sink} \theta \sin \omega_{i k} \mathrm{t}$.

Standing waves are characterized by the fact that their nodal diameters are fixed, which is evident from $(3,4)$. But since the original equation of free acoustic oscillations is linear, the sum and difference of its solutions in the form of standing waves $(3,4)$ are also its solutions. These solutions will accordingly be:

$\mathrm{A}(\mathrm{r})\left(\operatorname{cosk} \theta \cos \omega_{i k} \mathrm{t}+\sin \mathrm{k} \theta \sin \omega_{i k} \mathrm{t}\right)=\mathrm{A}(\mathrm{r})$ $\cos \left(\mathrm{k} \theta-\omega_{i k} \mathrm{t}\right)=\mathrm{A}(\mathrm{r}) \cos \mathrm{k}\left(\theta-\omega_{i k} / \mathrm{k} \mathrm{t}\right)$,

$\mathrm{A}(\mathrm{r})\left(\operatorname{cosk} \theta \cos \omega_{i k} \mathrm{t}-\operatorname{sink} \theta \sin \omega_{i k} \mathrm{t}\right)=\mathrm{A}(\mathrm{r})$ $\cos \left(\mathrm{k} \theta+\omega_{i k} \mathrm{t}\right)=\mathrm{A}(\mathrm{r}) \cos \mathrm{k}\left(\theta+\omega_{i k} / \mathrm{k} \mathrm{t}\right)$.

The expressions obtained are interpreted already by rotating waves, which are a particular case of traveling waves for bodies with cyclic symmetry. They are characterized in that their nodal diameters, just like natural forms itself at all (spatial amplitudes of vibrational displacements, velocities, pressures, etc.), rotate with their natural angular velocity $\omega_{i k} / \mathrm{k}$, at that in the counter directions. The first expression describes the rotation of the natural wave in the direction of increasing the angle $\theta$, while the second expression - in the opposite direction. We come therefore to the concept of a rotating resonant wave excited with an external rotating load, whose angular velocity $\omega$ approaches its natural angular velocity $\omega_{i k} / \mathrm{k}$. The forced wave rotation direction is given by the direction of rotation $\omega$ of the external load.

\section{Maps of dimensionless resonant angular velocities of hydroacoustic oscillations in a ring domain}

Thus, the circular shape of the geometric domain of an acoustic resonator provides a double multiplicity of its eigenfrequencies, i.e. each natural frequency $\omega_{i k}$ corresponds to the pair of eigenmodes, shifted along the angular coordinate by $\pi / 2 / \mathrm{k}$. Their superposition just gives a rotating natural form of vibration with its natural angular velocity $\omega_{i k} / \mathrm{k}$, which is excited by an external load rotating with the same angular velocity $\omega$ at the resonant mode of operation of the device.

Therefore, maps of dimensionless resonant angular velocities $\omega_{i k} / \mathrm{kR} / \mathrm{c}$ as a function of $\rho, 0 \leq \rho<1$ (Fig. 6-9) are of fundamental importance. Note that the introduction of the relative resonant angular velocity $\omega_{i k} / \mathrm{kR} / \mathrm{c}$ unifies the solution for any radii of circular concentric domains and working media within them by reducing the number of dimensional variables and giving an universal character to the above-mentioned maps. In the theory of similarity, which is the basis of modeling, the dimensionless quantities $\rho$ and $\omega_{i k} / \mathrm{kR} / \mathrm{c}$ are called similarity criteria, namely, $\rho$ is the geometric similarity criterion and $\omega_{i k} / \mathrm{kR} / \mathrm{c}$ is the physical similarity criterion for homochronicity known as the Strouhal number. The equality of all the same criteria of similarity for any system that obeys a particular physical phenomenon is a necessary and sufficient condition for the physical similarity of these systems [4]. This is what determines the universality of the maps of resonant angular velocities. 
The complete map of resonant angular velocities (Fig. 6) shows that:

1. The exact equality is performed for all dimensionless predominantly tangential angular velocities of the rotating waves in the resonance chamber at $\rho \rightarrow 1: \omega_{i k} / \mathrm{kR} / \mathrm{c}=1$, where $\mathrm{i}=1 ; \mathrm{k}$ is a natural number having the physical meaning of the number of nodal diameters of the proper form of predominantly tangential oscillations (Fig. 5).

2. All predominantly tangential velocities $\omega_{i k} / \mathrm{kR} / \mathrm{c}, \mathrm{i}=1 ; \mathrm{k}=1,2,3, \ldots$ for all values of $\rho, 0<\rho<1$ are assembled into a selfisolating single low-frequency branch, at which the device operates, whereas the predominantly radial velocities fill the entire high-frequency region very much densely.

To fine tune the device to the family of predominantly tangential resonant waves, it is necessary to refer to a larger scale midrange map (Fig. 7). It shows that the low-frequency branch of the predominantly tangential angular velocities of the rotating waves $\omega_{i k} / \mathrm{kR} / \mathrm{c}, \mathrm{i}=1$; $\mathrm{k}=1,2,3, \ldots$ for small values of $\rho$ splits into a dense bundle of nearby curves for different numbers of nodal diameters $\mathrm{k}$, whose density increases with increasing $\rho$, merging into a single line as $\rho$ increases further. Wherein the curve $\omega_{1 k} / \mathrm{kR} / \mathrm{c}$ at $\mathrm{k}=1$ bounds from above a family of predominantly tangential rotating resonant waves which are successively adjacent to it, decreasing with further growth of k, i.e. with sequential addition of the next nodal diameter in the corresponding rotating natural form.

Finally, low-frequency maps of rotating resonant waves give a detailed representation of the problem under investigation (Fig. 8). They show that the thinner the thickness of the annular resonator chamber, the higher the density of its natural angular velocities $\omega_{1 k} / \mathrm{kR} / \mathrm{c}$ and the greater the number of them that enters the near-resonance region when the chamber is excited by an external rotating acoustic wave with an angular velocity equal to $\omega_{11} / \mathrm{kR} / \mathrm{c}$. All natural angular velocities form a beam of rays radiating from a point on the map with coordinates: $\rho=1, \omega_{i k} / \mathrm{kR} / \mathrm{c}=1$ in the direction of decreasing abscissa $\rho$. With decreasing $\mathrm{k}$, the velocities $\omega_{i k} / \mathrm{kR} / \mathrm{c}$ increase. For $\mathrm{k}=8,7,1$ the cone of rays converging in the region of thin thicknesses of the resonator chamber is depicted in Fig. 9. The main conclusion from the analysis of resonant rotating waves is that absolutely all natural angular velocities for predominantly tangential waves $\omega_{i k} / \mathrm{kR} / \mathrm{c}, \mathrm{i}=1 ; \mathrm{k}=1,2,3, \ldots$ fall into the near-resonant area with a single unique resonance center $\omega_{i k} / \mathrm{kR} / \mathrm{c}=1$. Fig. 9 presents this unique feature in details. Consequently, there exists an unique angular velocity of the external acoustic load, which is a characteristic of the geometrical and physical parameters of the device, causing a simultaneous resonance of all predominantly tangential natural rotating waves. 


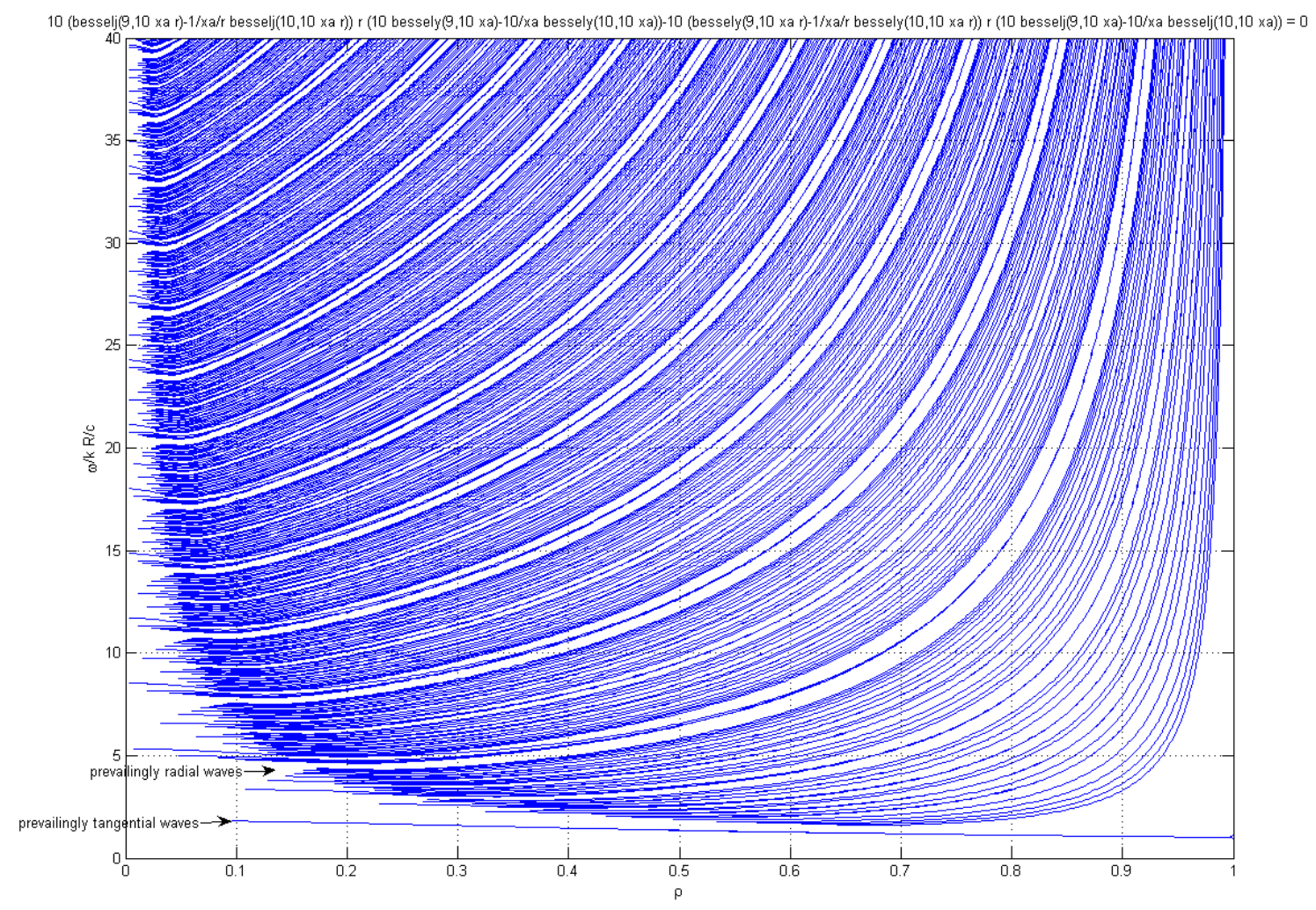

Figure 6. Small scale resonant angular velocity map.

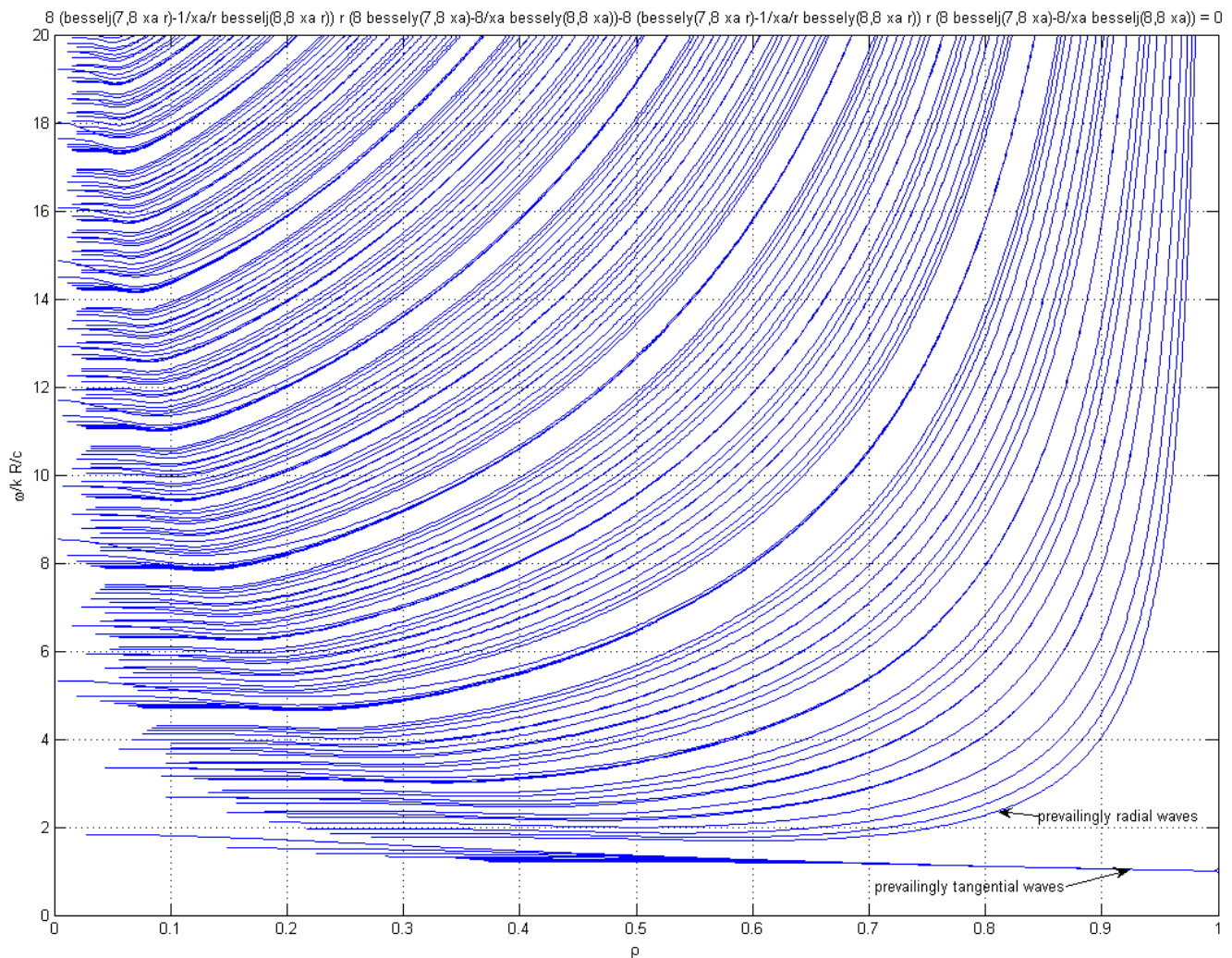

Figure 7. Medium scale resonant angular velocity map. 


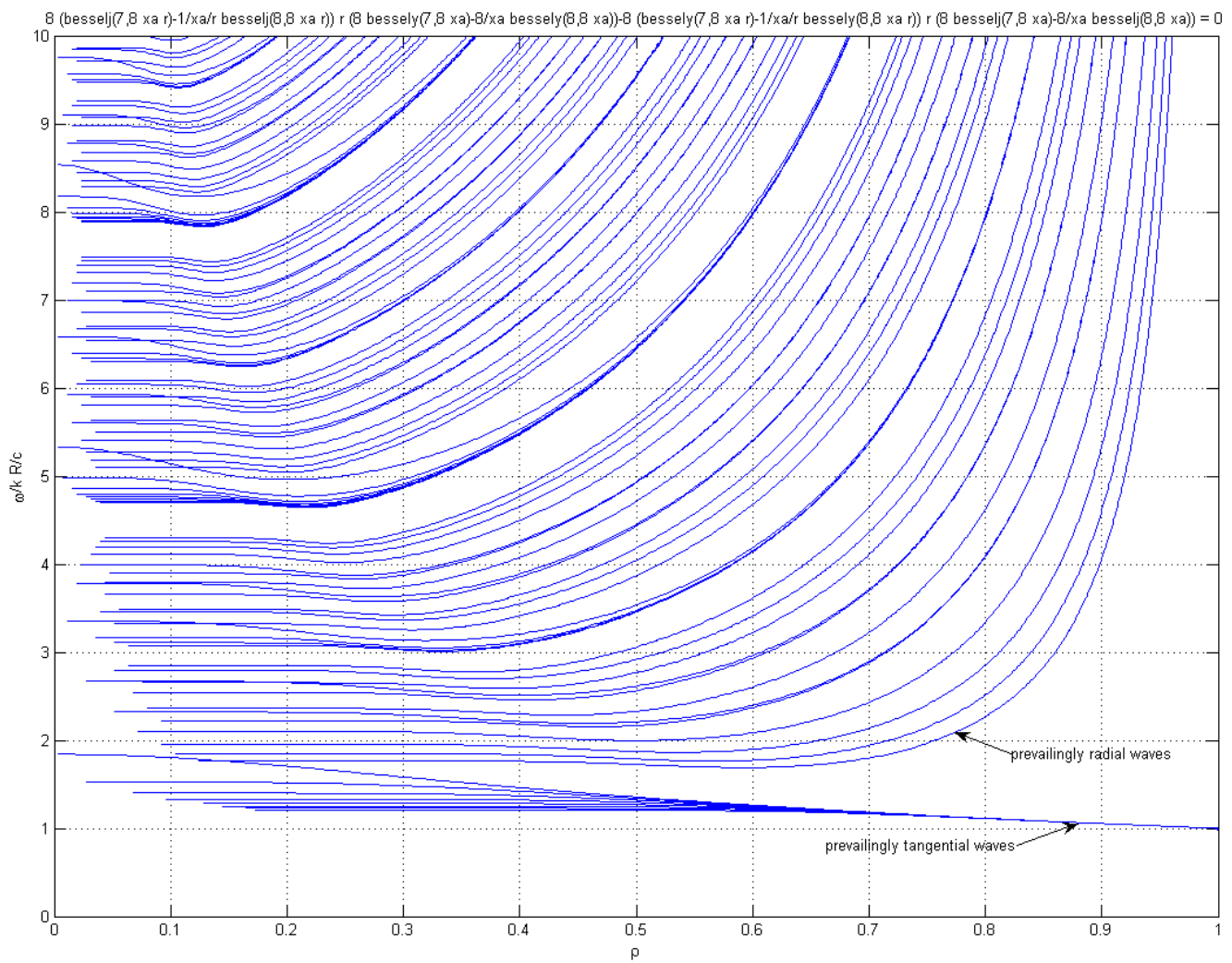

Figure 8. Large scale resonant angular velocity map.

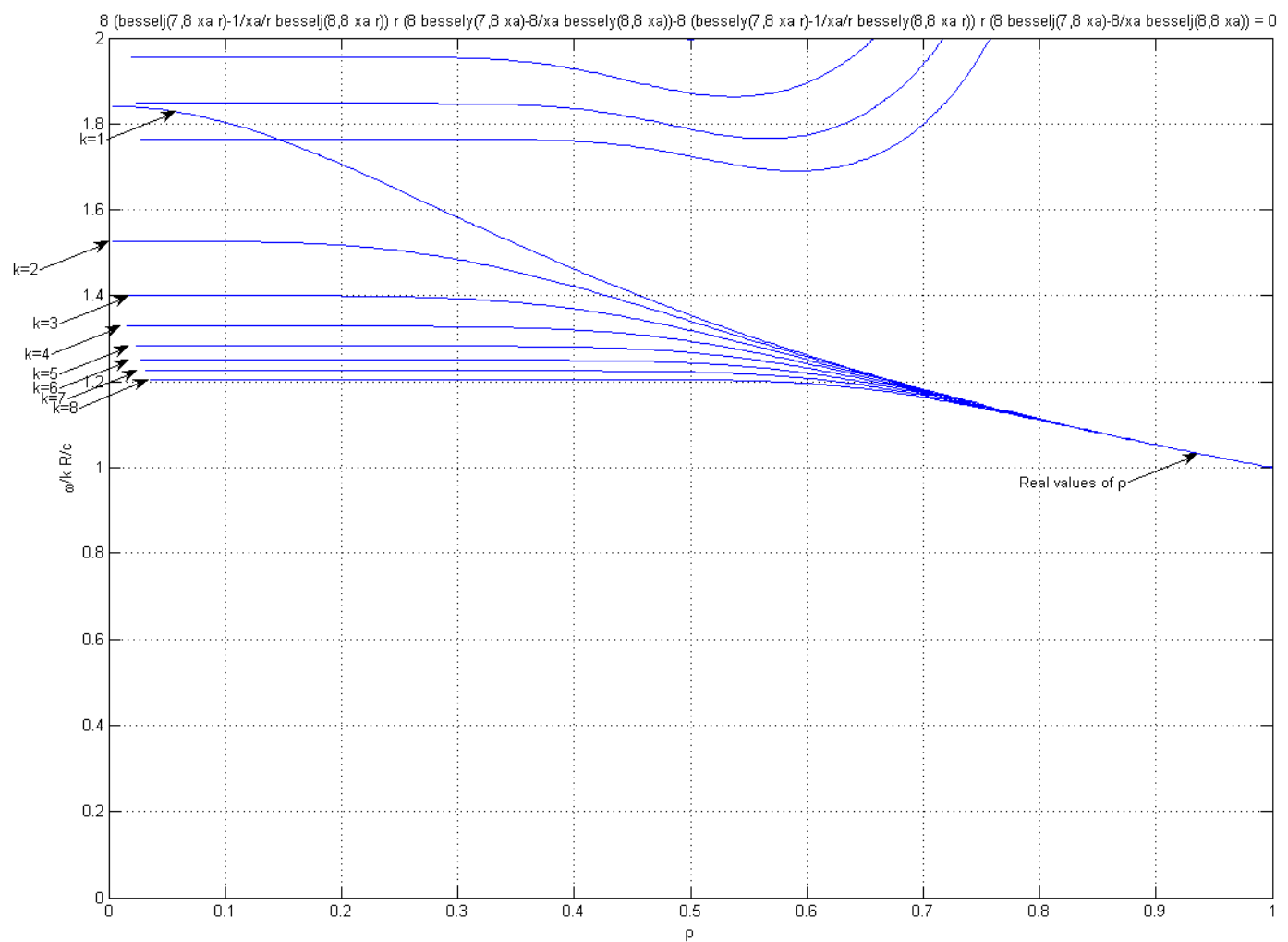

Figure 9. Resonant angular velocities for an annular domain. 


\section{Conclusions}

The use of ring resonators in nanotechnology is very beneficial due to the collection of all resonant rotating velocities into a single unique curve for all thickness values. Therefore, if the external load rotates with an angular velocity $\omega$ close to any natural angular velocity $\omega_{i k} / k$, then all resonant rotating waves are excited simultaneously. Thus, by tuning the angular velocity of the external rotating acoustic load to a single natural angular velocity, we automatically obtain a resonant excitation of all rotating natural forms.

\section{References:}

[1] http://www.rusnano.com/

[2] I.I. Bleckman, Vibrational Mechanics. Nonlinear Dynamic Effects, General Approach, Applications, World Scientific, Singapore, 2000.

[3] E.D. Sviyazheninov // RU Patent 2361683.

[4] L.I. Sedov, Similarity and Dimensional Methods in Mechanics, CRC Press, Boca Raton, 1993.

[5] V. Stojanović, Z. Milivojević, M. Praščević The Effect of Occupancy on Acoustic Parameters in a Concert Hall, WSEAS Transactions on Acoustics and Music, Volume 6, 2019, pp. 23-30

[6] Benoit Beckers, Omnidirectional Source Modeling for Ray Tracing in Room Acoustics with Specular Reflection, WSEAS Transactions on Acoustics and Music, Volume 6, 2019, pp. 13-22

[7] Lamberto Tronchin, The New Teatro "Amintore Galli" in Rimini: Acoustic Design and Measurements on Diffusing Panels, WSEAS Transactions on Acoustics and Music, Volume 6, 2019, pp. 1-6 